\title{
Modelling of Cosmic Molecular Masers: Introduction to a Computation Cookbook
}

\author{
Andrej M. Sobolev ${ }^{1}$ and Malcolm D. Gray ${ }^{2}$ \\ ${ }^{1}$ Ural Federal University, \\ Lenin Ave. 51, Ekaterinburg 620000, Russia \\ email: Andrej.Sobolev@usu.ru \\ ${ }^{2}$ University of Manchester, Manchester M13 9PL, UK \\ email: Malcolm.Gray@manchester.ac.uk
}

\begin{abstract}
Numerical modeling of molecular masers is necessary in order to understand their nature and diagnostic capabilities. Model construction requires elaboration of a basic description which allows computation, that is a definition of the parameter space and basic physical relations. Usually, this requires additional thorough studies that can consist of the following stages/parts: relevant molecular spectroscopy and collisional rate coefficients; conditions in and around the masing region (that part of space where population inversion is realized); geometry and size of the masing region (including the question of whether maser spots are discrete clumps or line-of-sight correlations in a much bigger region) and propagation of maser radiation. Output of the maser computer modeling can have the following forms: exploration of parameter space (where do inversions appear in particular maser transitions and their combinations, which parameter values describe a 'typical' source, and so on); modeling of individual sources (line flux ratios, spectra, images and their variability); analysis of the pumping mechanism; predictions (new maser transitions, correlations in variability of different maser transitions, and the like). Described schemes (constituents and hierarchy) of the model input and output are based mainly on the experience of the authors and make no claim to be dogmatic.
\end{abstract}

Keywords. interstellar matter, molecules, masers, numerical modelling

\section{Introduction}

Creating maser models makes it possible to justify the use of masers as sensitive tracers of specific objects (young stellar objects, late-type stars, active galactic nuclei, for example), to use them as tools (to study kinematics, measure physical parameters including magnetic fields, to make direct measurements of distance, and to study the structure of the Galaxy, to name just some), and to study masers as a unique phenomenon which is interesting in itself.

Modelling allows us to find likely/optimum conditions in the maser sources, interpret spectra and maps of generic sources and individual objects, reveal relations between maser spots and physical condensations, understand variability, predict likely new masers, reveal detailed pumping schemes and resolve propagation problems and so on.

In many cases modelling of cosmic molecular masers should be numerical. Analytical estimates often can carry only qualitative character for at least two reasons. The first reason is connected with the nature of the maser phenomenon, and in particular the complexity of the pumping and saturation schemes in real molecules: maser amplification happens due to the existence of the population inversion which is created by the pumping mechanism. For the brightest masers it was shown that these mechanisms involve very large numbers of levels and transitions (see, for example, Sobolev (1989) for $\mathrm{H}_{2} \mathrm{O}$ masers, Sobolev \& Deguchi (1994a) for $\mathrm{CH}_{3} \mathrm{OH}$ masers and Gray (2007) for $\mathrm{OH}$ 
masers). Typically the figures are hundreds of levels and thousands of transitions. We note that here, and further on, we are trying to cite the historically earliest papers in accessible journals; later works refer to them. Given the likely numbers of levels and transitions, analytical techniques must almost always give way to the processing power of the computer. The second reason is connected with the complexity of the astrophysical maser environments. Simple geometrical models can describe these systems only approximately. The presence of inversions itself requires non-equilibrium conditions, where there is an interplay between different constituents of the matter and radiation, magnetic and velocity fields. The masing gas is often subject to large-scale velocity gradients, and the medium is normally highly turbulent at smaller scales. For some specific examples, see Deguchi (1982) and Humphreys et al. (1996) for circumstellar masers, Sobolev, Wallin \& Watson (1998) for interstellar masers and Wallin, Watson \& Wyld (1998) for masers in the accretion disks of active galactic nuclei. Additionally, in some cases, maser amplification can be restricted by the capacity of the medium to carry emission of extreme brightness (the phenomenon known as saturation) and extremely bright emission affects the medium through which it propagates.

In this paper we will try to outline the basics of computational modelling of astrophysical molecular masers in the most widespread case of stationary, that is steady-state, masers which arise due to a pumping mechanism creating inversion of population numbers for the levels of one or more maser transitions.

\section{Model input}

The first essential stage of model construction is assembly of all the relevant input data and relations that are necessary for construction of a computational model.

Scheme of levels. Astrophysical molecular masers appear because of the operation of the pumping mechanism. In the majority of cases, this consists of the combined action of numerous pumping cycles. Links of these cycles connect energy levels of the molecule and correspond to quantum-mechanical transitions, usually driven by radiative and/or collisional processes. It is very important to know which levels are essential for operation of the pumping mechanism. This often requires special consideration, based on the knowledge of relevant molecular spectroscopy and physical conditions in the maser source and its environment which in turn controls the transition rates. It is generally thought that the scheme of energy levels used for computations can be limited to those levels which are expected to have high population numbers (numbers of molecules in the state corresponding to the particular energy level). In some cases this can be erroneous, and it is recommended to include also levels which are connected with the highly populated levels by the set of transitions of high probability (that is possessing large radiative or collisional rates). This statement is illustrated by the example of class II methanol maser pumping where the sparsely populated torsionally excited levels play the role of extremely important transients: in computations of Sobolev \& Deguchi (1994b), neglecting torsionally excited levels in a representative model turned strong maser emission into absorption. It is difficult to make recommendations on how to construct adequate schemes of levels for the cases when available molecular spectroscopy data is insufficient. But for the case of sufficient spectroscopic data one can constrain an adequate scheme of levels using an approach similar to described in Sobolev (1989) and Sobolev \& Deguchi (1994b).

Transition rate coefficients. The first numerical models appeared just after reliable estimates for the rates of transitions became available (see de Jong (1973), Shmeld et al. (1976) and Bolgova et al. (1988) for different pumping mechanisms of interstellar $\mathrm{H}_{2} \mathrm{O}$ 
masers, Deguchi (1977) for circumstellar $\mathrm{H}_{2} \mathrm{O}$ masers, Cesaroni \& Walmsley (1991) and Gray et al. (1991) for OH masers in various sources of different nature, Sobolev \& Strelnitskii (1983) and Sobolev \& Deguchi (1994b) for $\mathrm{CH}_{3} \mathrm{OH}$ masers of different classes, Deguchi \& Iguchi (1976) for circumstellar SiO masers). It is worth pointing out that the absence of essential data on molecular excitation forced early enthusiasts of maser research to use (and sometimes invent) approximations for the necessary values. Most of the papers mentioned above used different guesses on the rules and values of collisional rates. These guesses were not purely serendipitous and were based on deep knowledge of molecular spectroscopy and physics of collisional excitation. Moreover, stability of conclusions was usually verified by comparison of results obtained with different models for the transition rates. Later examination (see for example Cragg et al. (2005) for $\mathrm{CH}_{3} \mathrm{OH}$ maser models) with the use of refined data have shown that the qualitative conclusions of the previous modelling remained unchanged, though some numbers, for example the density threshold for collisional quenching of class II $\mathrm{CH}_{3} \mathrm{OH}$ masers, altered by an order of magnitude.

Conditions in and around masing regions. The next essential part of the input consists of a description of the physical parameters in the masing region (internal conditions), and in its nearby environment (external conditions). Important parameters determining internal conditions include the kinetic temperature, abundance of the maser molecule and number density of the medium - all of which affect collisional and radiative rates - and the velocity field, and the temperature of any dust present, which affect radiation transfer specifically. Examples of the second (external) type are usually restricted to radiation fields (typically from a nearby protostellar object in the case of a star-forming region maser). Population transfer rates are usually represented as products of a (first-order) rate-coefficient and a fractional level population. For the case of collisional rates, the first order rate coefficient is in turn a product of the number density of a collision partner and a second-order rate coefficient. The functional forms of such coefficients are relatively well known (see, for example, the relevant theory in Flower (2003)), and are normally determined by the kinetic temperatures of collisional partners, usually molecular hydrogen and helium (see, e.g. Cragg et al. (2005) for the models of class II $\mathrm{CH}_{3} \mathrm{OH}$ masers). In some cases other essential collisional partners (e.g. electrons in Bolgova et al. (1988)) were introduced. In contrast, functions of physical parameters associated with the radiative rates are very complicated, and usually require long computations. This comes about because evaluation of radiative rates requires solution of the radiative transfer problem, the complexity of which will be discussed in the special subsection below. Radiative rates are determined by emission from external and internal sources and by extinction (absorption and scattering) within the region where the maser is formed. Knowledge of the radiation field parameters is required for all important pumping lines in addition to the maser line itself. Here, we provide some explanatory notes on the physical parameters that determine the effective rate coefficients of radiative fields. Emission and absorption in transitions of the masing molecule itself were shown to be important by calculations in some of the earliest models (see, for example, de Jong (1973)). In the simplest and most common case, the pumping is realized via collisional excitation and radiative decay, followed by escape of the photons from the maser formation region. Later it was established that absorption by internal dust can create a maser even in the case where high optical depth in the pumping lines, within the object, prevents escape of photons (see, for example, Shmeld et al. (1976)). In some cases, this internal dust plays the role of a source of energy for the pumping, as in Voronkov et al. (2005), so the role of internal dust is twofold. Dust emission from the maser environment can be the main source of energy in the pumping mechanism. Good examples of this are presented by the strong class II 
methanol masers and OH masers (Sobolev \& Deguchi (1994b), Cragg et al. (2005) and Gray (2001)). It is important to mention that the properties of the dust grains (sizes, composition, an so on) influence its spectrum. These differences can affect maser line intensities considerably (see Ostrovskii \& Sobolev (2002), Gray (2001)). Apart from the dust, the role of external sources of pumping radiation can be taken by optically thick HiI regions (Slysh et al. (2002)). This is most efficiently done by the youngest sources, hypercompact His regions, which are bright at sufficiently high frequencies (Sobolev et al. (2007)). External sources of radiation also play an important role as the background that is amplified by the masers (Cooke \& Elitzur (1985), Sobolev \& Deguchi (1994b) and Gray (2001)). It should be noted that the maser brightness is a nonlinear function of the background intensity. It was shown that in some cases background emission that gives considerable radiation density at the frequency of a maser transition can produce maser saturation effects (see Sobolev \& Deguchi (1994b) and Sobolev et al. (1997a)). The last parameter of the maser formation region which we would like to mention here has a chemical nature. It is the molecular abundance of the maser molecule, which is very important because it determines number of masing molecules in the line of sight, and, hence, the brightness of the maser emission. Chemical questions are very important because explaining the strongest masers often requires an assumption of a very high abundance of the masing molecule. Such high abundances put restrictions on the physical state and evolution of the region. Chemical considerations are also very important when one considers relative intensities of masers in transitions of different maser species (see the analysis of coexistence of $\mathrm{OH}$ and $\mathrm{CH}_{3} \mathrm{OH}$ masers in Hartquist et al. (1995) and Cragg et al. (2004)).

Geometry, kinematics and size of the masing region. The geometry of the maser region strongly affects the solution of the radiative transfer problem in both its aspects: calculation of internal radiative fields (the necessity of which were considered in the previous subsection) and calculation of the properties of the emergent radiation. The geometry of masing objects at the scale of, say, VLBI resolution is usually difficult to establish. However, on the larger scale of a formation region, consisting of many individual masing objects or features, it is often possible to suggest a suitable geometry for modelling, often from the evidence of observations of the source in non-maser lines, particularly in lines of $\mathrm{CO}$, that trace low-velocity molecular outflows, and in lines of shock-tracing molecules, for example $\mathrm{SiO}$ and molecular hydrogen. There are various forms commonly used for modelling: megamasers typically have an overall disk or toroidal structure (Lockett \& Elitzur (2008) and the clumpy model by Wallin et al. (1998)), and a similar geometry is applicable to some types of star-forming region masers (see, for example, the model in Gallimore et al. (2003)). Other star-forming region masers have been placed at the edges of an outflow, as in models by Mac Low et al. (1994) and Sobolev et al. (1998)). Masers associated with shocks, for example $\mathrm{OH}$ 1720-MHz masers in SNRs and, in particular, water masers in outflows are often treated in a slab geometry with the slabs arranged parallel to the shock front (for example, Yates et al. (1997)). For masers associated with the shell at the edge of an Hir region, slab models have also been used, but introducing clumps is sometimes necessary (Sutton et al. (2001)). For stellar masers, the obvious geometry is spherical, since these masers form in shells in the circumstellar envelope. However, for certain supergiants and post-AGB objects, the spherical approximation is poor, and the model should probably rely on a different geometry including clumps (for example Humphreys et al. (1996)). Processes of radiative transfer are tightly related to velocity fields within the region. It is well known that monotonic large velocity gradients lead to localization of the radiative transfer problem, which simplifies calculations and reduces the number of molecules contributing to maser emission. Non-monotonic and 
irregular fields result in interactions between spatially differentiated parts of the object which greatly affects maser spectra and images, as in Deguchi (1982), Humphreys et al. (1996) and Sobolev, Wallin \& Watson (1998). Velocity fields cause non-local line overlaps which can play an extremely important role in the pumping of some maser species (see OH maser models in Cesaroni \& Walmsley (1991) and Pavlakis \& Kylafis (1996)). It should be noted that the observational data on maser spot kinematics (radial velocities and proper motions) provides tight constraints for the computational models, but only in rare cases does it provide definitive association with a certain type of astrophysical object, such as the accretion disk in NGC 4258 described in Greenhill et al. (1995), or the outflow in the BN/KL region in Orion, described in Greenhill et al. (1998). Often interpretation is not unique (see the discussion of the disk versus outflow model in Shchekinov \& Sobolev (2004) and various models in van der Walt et al. (2007)). Combined computations of kinematics and excitation are necessary to distinguish between the various possibilities.

Discrete clumps or correlations? We would like to draw attention to one basic problem relevant to the geometry and size of the maser formation region. The question is often polarized and reduced to two extreme cases when 1) maser spots are assumed to be discrete clumps (density inhomogeneities) with sizes comparable to the sizes of the maser spots, and 2) maser spots are assumed to be correlations in the distributions of some physical parameters within an orders of magnitude more extended maser formation region (possibilities include correlations in velocity fields suggested in Deguchi (1982) and Sobolev, Wallin \& Watson (1998) or alignment of the structures in the line of sight suggested in Deguchi \& Watson (1989)). The importance of this question is directly related to interpretation of the motion of maser spots and trigonometric parallax measurements (see, for example, Bloemhof et al. (1996) and Sobolev et al. (2008)). Small angular sizes of maser spots, and normally clear distinction of the spots from each other, suggest that the maser spots are formed in discrete clumps. This view is supported by a number of observational facts. For example, the ordered angular and spatial spot distribution, and the orientation in general agreement with the proper motions, support a kinematical interpretation for velocities of the $6-\mathrm{GHz} \mathrm{CH}_{3} \mathrm{OH}$ maser spots in several sources (see, for example, Moscadelli et al. (2011)). The correlation interpretation is supported by the detection of extended maser structures, with sizes orders of magnitude greater than those of the maser spots themselves (such observations are described in Gwinn (1994), Minier et al. (2002), Harvey-Smith \& Cohen (2006) and interpretations in Sobolev et al. (2003)). Observations indicate that there can be numerous maser spots within one extended maser structure. It is instructive to consider the situation in the context of such a well-studied region as W3(OH). Bloemhof et al. (1996) measured intrinsic morphologies of interstellar $\mathrm{OH}$ maser spots in this source at two distinct epochs. They found that maser spot shapes persist with time, and came to the following conclusion: 'These observations provide the first direct evidence that the motions measured are due to actual physical movement of discrete clumps of maser-emitting matter, rather than to some sort of nonkinematic effect, such as traveling excitation phenomena or chance realignments of coherency paths through the masing gas. The kinematic assumption is crucial to astrophysical applications of maser proper-motion measurements, including distance determinations and studies of source dynamics.' Xu et al. (2006) measured trigonometric parallaxes for numerous $6-\mathrm{GHz} \mathrm{CH}_{3} \mathrm{OH}$ maser spots, and have found that the measured values are in close internal agreement. This provides support to the kinematic (discrete clump) hypothesis because the $6-\mathrm{GHz}_{3} \mathrm{CH}_{3} \mathrm{OH}$ masers in $\mathrm{W} 3(\mathrm{OH})$ are distributed within the same region as those of $\mathrm{OH}$ (Menten et al. (1988)). At the same time, considering BIMA data on the numerous maser and non-maser $\mathrm{CH}_{3} \mathrm{OH}$ lines in W3(OH) Sobolev 
et al. (2005) concluded that the $6-\mathrm{GHz} \mathrm{CH}_{3} \mathrm{OH}$ maser formation region in this source has a size of order 1 arcsecond, which is about 3 orders of magnitude greater than that of the $6.7-\mathrm{GHz}$ maser spots, and corresponds to the total extent of the region where the strong class II methanol masers are distributed. This conclusion was later supported by the observational discovery of large-scale methanol and hydroxyl maser filaments in W3(OH) (Harvey-Smith \& Cohen (2006)). Computational modelling has shown that the model comprising a uniform masing layer and a turbulent velocity field (that is spots appear due to velocity correlations) has good potential to reproduce observational data following the time evolution of the maser images (Sobolev et al. (2003)), and does not prevent measurements of trigonometric parallaxes (Sobolev et al. (2008)). Of course these models are greatly simplified but they have shown the potential of the correlation interpretation. The actual situation is likely to be in between these extremes, and the maser images and spectra, as well as their evolution, are probably determined both by relatively well-delineated clumps (density inhomogeneities) and correlations (coincidences of velocities and other physical parameters in the line of sight). In conclusion, though at present this complicated situation cannot be resolved both by observational and computational means, it should be noted that the kinematic interpretation of the maser spot velocities should not be considered as contradictory to existence of correlations within extended masing regions.

Radiative transfer. The solution to any one model in a parameter-space search consists of a set of population numbers for the masing molecule at each discretized position within the model geometry. For example, in a uniform spherical model, the solution is completely specified by one set of population numbers at each modeled radius. To obtain such a solution requires solving the combined radiative transfer and kinetic master (or statistical equilibrium) equations. The majority of models have used different methods that allow us to localize the radiative transfer problem (see, for example, de Jong (1973), Shmeld et al. (1976) and Deguchi \& Iguchi (1976)). The large velocity gradient (LVG) approximation was most widely used. The geometry in it is apparent only through the form of the LVG escape probabilities that have to be solved for, with the population numbers, at a single representative point. Self-consistent population numbers and escape probabilities are achieved iteratively. More sophisticated models typically rely on a radiative transfer algorithm such as accelerated lambda iteration (ALI) or coupled escape probability (CEP) to provide sets of populations with spatial resolution. The ALI method as applied to molecular line transfer (Scharmer \& Carlsson (1985)) relies on a solution of modest accuracy from ordinary lambda-iteration, that is an iterative solution of the radiative transfer and kinetic master problems from a starting solution, typically with Boltzmann population numbers. The ALI theory introduces perturbations on all population and radiative variables, and constructs a set of equations for these using an approximate, usually local, lambda operator. These perturbations are then iterated to zero against an error vector based on the exact lambda operator. ALI has the advantage of only ever having to solve linear equations, but it remains necessary to compute various radiation integrals over frequency and direction. This method was successfully applied to the interpretation of the $\mathrm{OH}$ masers (Gray (2001)). It is available in slab, spherical and perhaps other geometries. The Monte Carlo approach was suggested by Bernes (1979). A great advantage of this method is the possibility of solving radiative transfer problems in the maser and pumping lines simultaneously. It was applied to interpret the $25-\mathrm{GHz}$ $\mathrm{CH}_{3} \mathrm{OH}$ masers in Orion by Sobolev \& Strelnitskii (1983). This method is applicable for any geometry (see, for example, Juvela (1998)), but requires great computational time. This is the main reason why this method has not been widely used for maser modeling. There has been recent renewed interest in integral equation (direct non-linear) 
methods, and particularly the CEP implementation (Elitzur \& Asensio Ramos (2006)). In such methods, the radiation mean intensity is eliminated analytically, yielding a set of integral equations in the unknown population numbers. In a discrete model, the integral equations become non-linear algebraic equations in the population numbers. A spherical version of the CEP algorithm has recently been implemented (Yun et al. (2009)). Acceleration of convergence of the complicated models introduced above can often be significantly improved by the use of convergence accelerators that use knowledge of past iterates to make an improved estimate of the most recent. Two accelerators worthy of attention are Ng's method (Ng (1974)) and the ORTHOMIN accelerator (Vinsome (1976)).

Propagation of maser radiation. The propagation of the maser radiation is directly related to the formation of images and affects their intensities. Even in the simplest models, where the maser radiation is unsaturated and the radiative transfer problem is not fully resolved, this can require considerable computational effort (see the models of the turbulent medium in Sobolev, Wallin \& Watson (1998) and the circumstellar envelope in Humphreys et al. (1996)). Further, the propagation of the maser radiation introduces additional problems that do not usually apply to the transfer of, for example, the pumping radiation in the same model. One of these is that many radiative transfer codes are not constructed to function properly when the optical depth and source function become negative, as they will for masers. There is also the geometrical problem that the maser radiation is tightly beamed, so that a better angular resolution is required for the maser rays than for the rays of the non-maser radiation. A more fundamental consideration is that maser radiation is expected to retain some coherence properties that cause a departure from the Gaussian statistics of ordinary radiation. To model this behaviour correctly, maser saturation must be considered in the semi-classical approximation, where the molecular response is treated quantum-mechanically through density-matrix theory. Semi-classical models of an ideal 1-D maser with full semi-classical coupling have been considered by Menegozzi \& Lamb (1978), and in the more recent work by Dinh-v-Trung (2009a); Dinh-v-Trung (2009b), where the latter work includes polarization. An approximate semi-classical model that allows propagation of the power spectrum, rather than many realizations of the electric field has also been devised (Field \& Gray (1988)). Numerical solution of the full equations for the frequency-dependent radiative transport that includes the thermal motion of the molecules is very complicated. Until now it was carried out only in Watson \& Wyld (2003) for the simple geometries of isolated spheres and thin disks viewed edge-on. Another complication in computation of propagating maser radiation arises in the presence of magnetic fields causing splitting and polarization of the maser lines. This is especially important for $\mathrm{OH}$ masers, modelled in Watson et al. (2004).

\section{Model output}

The outcome of the modelling has different forms depending on the task of research study. It can be exploratory or address some well-defined question. This section describes some types of model output used in our research.

Exploration of parameter space. One of the primary tasks of the modelling is search for the ranges of parameters where the model can reproduce observed values of maser fluxes, flux ratios (when applicable) and other characteristics. The goal is achieved by exploration of a parameter space which can be done in several different ways. Model calculations can require considerable computer time. To gain a broad view, it is useful to study the effects on the output parameters of varying certain of the model input parameters one-at-a-time about chosen standard conditions. The other model parameters remain 
fixed at the standard values in all runs. This approach was used in, among other works, Pavlakis \& Kylafis (1996) and Sobolev et al. (1997a) with considerable success. However, in some cases choice of a single or even a few representative models is not possible, and it is necessary to explore wide ranges of parameter space. In such a situation it is common to calculate a grid of models with relevant detailing. The number of maser model parameters usually exceeds 3 , and presentation of results in the form of cross-sections looks adequate. Rather sophisticated ways of presentation of 3D cross-sections can be found, for example, in Juvela (1998). In many cases conciseness, combined with sufficient clarity can be achieved following the style of presentation used in Cragg et al. (2002), where the brightness temperature was shown as a set of contours in a set of cross-sections with coordinates representing 2 parameters (hydrogen number density and specific column density of the masing molecule). Exploration of the parameter space is used when it is necessary to constrain parameter domains of a generic source that represents common characteristics of some masing object (Cragg et al. (2004)). In other circumstances, it may be preferable to create models of individual objects (next subsection), or to study pumping regimes (see example in Sobolev et al. (2007)).

Modelling of individual sources. Creating models of the most-studied and prototypic sources is one of the basic tasks of computational modelling. The models are designed to explain one or more sets of observational data. One type of modelling studies is devoted to searching for the set of parameters providing the best (or at least an acceptable) fit to observational data. Many computational models based on pumping analysis succeeded in reproducing observed line flux ratios of individual objects $\left(\mathrm{CH}_{3} \mathrm{OH}\right.$ masers in $\mathrm{W} 3(\mathrm{OH})$ by Sutton et al. (2001), G345.01+1.79 and NGC 6334F by Cragg et al. (2001), OH masers in the magnetic-field specific model of W75N by Gray et al. (2003)). Other models managed to obtain fits to maser kinematics $\left(\mathrm{H}_{2} \mathrm{O}\right.$ masers in $\mathrm{W} 49 \mathrm{~N}$ by Mac Low et al. (1994) and in L1287 by Fiebig (1997)). Another type of modelling studies aims to reproduce general characteristics of the observational data because searching for exact coincidence is impossible, for example because of the stochastic character of the emission. Reproducing spectra and images for $\mathrm{CH}_{3} \mathrm{OH}$ masers in Ori-KL was done by Sobolev et al. (1998), for $\mathrm{CH}_{3} \mathrm{OH}$ masers in $\mathrm{W} 3(\mathrm{OH})$ by Sobolev et al. (2005), and for SiO masers in o Cet by Gray et al. (2009). Maser variability was addressed in Sobolev et al. (2003) and Gray (2005).

Analysis of the pumping mechanism. Analysis of the nature of the maser phenomenon requires simulation of the pumping mechanism. As discussed above, such mechanisms consist of the combined action of numerous pumping cycles. Heating cycles deliver molecules from the excitation state corresponding to the lower energy level (briefly, lower maser level) of the maser transition, up to the upper maser level. Cooling cycles have opposite starting and final points. It is instructive to describe pumping as a heat engine as suggested in Strelnitskii (1988, and this volume). In this approach, transitions of the upward links of the pumping cycles represent the source of the pumping energy, and downward links provide the sink of energy. Numerical definition of the pumping cycle efficiency, and means to draw conclusions about the thermodynamic nature of these links were introduced in Sobolev (1986). This provided grounds for thermodynamic methods of analysis of the pumping mechanism based on degrees of participation of the cycles of certain thermodynamic types described in Sobolev et al. (1989), and for the methods of tracing pumping routes described in Sobolev \& Deguchi (1994a) and Gray (2007). These methods were successfully applied to analysis of the pumping of $\mathrm{CH}_{3} \mathrm{OH}$ masers (Sobolev \& Deguchi (1994b), Sutton et al. (2001)) and OH masers (Gray (2007, and this volume)). Examples of detailed analysis of behaviour of the characteristics of the 
pumping cycles for masers under different degrees of saturation is presented in Sobolev \& Deguchi (1994a).

Predictions. An attractive point in maser computations is their predictive potential. The papers Sobolev et al. (1997b) and Johns et al. (1998: substituted $\mathrm{CH}_{3} \mathrm{OH}$ species) were devoted to extraction of data on the maser candidates from a number of models of class II $\mathrm{CH}_{3} \mathrm{OH}$ masers. Some predictions were observationally confirmed later (for example Cragg et al. (2001), Sutton et al. (2001), Voronkov et al. (2002), and Sobolev et al. (2002)). It is noteworthy that some of the successful predictions did not follow the phenomenological series rules described in Menten (1991) and Sobolev (1993). The situation regarding predictions of class $\mathrm{I} \mathrm{CH}_{3} \mathrm{OH}$ maser candidates is quite similar, and is described in more detail in the contribution by Voronkov et al. (this volume). Modelling also allows us to predict correlations in variability of different maser transitions and their response to evolution of the pumping conditions (see, for example, Gray (2005)).

\section{Acknowledgements}

AMS thanks the Russian Foundation for Basic Research (grant 10-02-00589-a) and the Russian federal task program 'Research and operations on priority directions of development of the science and technology complex of Russia for 2007-2012' (state contract 16.518.11.7074). The authors are grateful to V.S. Strelnitski for fruitful discussion.

\section{References}

Bernes, C. 1979, Astron. Astrophys., 73, 67

Bloemhof, E. E., Moran, J. M., \& Reid, M. J. 1996, ApJ, 467, 117

Bolgova, G. T., Makarov, S. V., \& Sobolev, A. M. 1988, Astrophysics, 28, 239

Cesaroni, R. \& Walmsley, C. M. 1991, Astron. Astrophys., 241, 537

Cooke, B. \& Elitzur, M. 1985, ApJ, 295, 175

Cragg, D. M., Sobolev, A. M., Ellingsen, S. P., et al. 2001, MNRAS, 323, 939

Cragg, D. M., Sobolev, A. M., \& Godfrey, P. D. 2002, MNRAS, 331, 521

Cragg, D. M., Sobolev, A. M., Caswell, et al. 2004, MNRAS, 351, 1327

Cragg, D. M., Sobolev, A. M., \& Godfrey, P. D. 2005, MNRAS, 360, 533

Deguchi, S. \& Iguchi, T. 1976, PASJ, 28, 307

Deguchi, S. 1977, PASJ, 29, 669

Deguchi, S. 1982, PASJ, 29, 669

Deguchi, S. \& Watson, W. D. 1989, ApJ, 340, 17

de Jong, T. 1973, Astron. Astrophys., 26, 297

Dinh-v-Trung 2009, MNRAS, 396, 2319

Dinh-v-Trung 2009, MNRAS, 399, 1495

Elitzur, M. \& Asensio Ramos, A. 2006, MNRAS, 365, 779

Fiebig, D. 1997, Astron. Astrophys., 758, 770

Field, D. \& Gray, M. D. 1988, MNRAS, 234, 353

Flower, D. R. 2003, Molecular Collisions in the Interstellar Medium, pp. 145. ISBN 0521545749. Cambridge, UK: Cambridge University Press, December 2003.

Gallimore, J. F., Cool, R. J., Thornley, M. D., \& McMullin, J. 2003, ApJ, 586, 306

Gray, M. D., Doel, R. C., \& Field, D. 1991, MNRAS, 252, 307

Gray, M. D. 2001, MNRAS, 324, 57

Gray, M. D., Hutawarakorn, B., \& Cohen, R. J. 2003, MNRAS, 343, 1067

Gray, M. D. 2005, Astrophys.Space Sci., 295, 309

Gray, M. D. 2007, MNRAS, 375, 477

Gray, M. D., Wittkowski, M., Scholz, M., et al. 2009, MNRAS, 394, 51

Greenhill, L. J., Jiang, D. R., Moran, J. M., et al. 1995, ApJ, 440, 619 
Greenhill, L. J., Gwinn, C. R., Schwartz, C., et al. 1998, Nature, 396, 650

Gwinn, C. 1994, ApJ, 431, L123

Hartquist, T. W., Menten, K. M., Lepp, S., \& Dalgarno, A. 1995, MNRAS, 272, 184

Harvey-Smith, L. \& Cohen, R. J. 2006, MNRAS, 371, 1550

Humphreys, E. L. M., Gray, M. D., Yates, J. A., et al. 1996, MNRAS, 282, 1359

Johns, K. P., Cragg, D. M., Godfrey, P. D., \& Sobolev, A. M. 1997, MNRAS, 300, 999

Juvela, M. 1998, Astron. Astrophys., 329, 659

Lockett, P. \& Elitzur, M. 2008, ApJ, 677, 985

Mac Low, M.-M., Elitzur, M., Stone, J. M., \& Koenigl, A. 1994, ApJ, 427, 914

Menegozzi, L. N. \& Lamb, Jr., W. E. 1978, Phys. Rev. A, 17, 701

Menten, K. M. 1991, ASPC, 16, 119

Menten, K. M., Johnston, K. J., Wadiak, E. J., et al. 1988, ApJ, 381, L41

Minier, V., Booth, R. S., \& Conway, J. E. 2002, Astron. Astrophys., 383, 614

Moscadelli, L., Sanna, A., \& Goddi, C. 2011, Astron. Astrophys., 536, 38

Ng, K.-C. 1974, JCP, 61, 2680

Ostrovskii, A. B. \& Sobolev, A. M., 2002, IAUS, 206, 183

Pavlakis, K. G. \& Kylafis, N. D. 1996, ApJ, 467, 300

Scharmer, G. B. \& Carlsson, M. 1985, J. Comp. Phys., 59, 56

Shchekinov, Yu.A. \& Sobolev, A. M. 2004, Astron. Astrophys., 418, 1045

Shmeld, I. K., Strel'nitskii, V. S., \& Muzylev, V. V. 1976, Soviet Astronomy, 20, 411

Slysh, V. I., Kalenskii, S. V., \& Val'tts, I. E. 2002, Astron. Reports, 46, 49

Sobolev, A. M. \& Strelnitskii, V. S. 1983, Soviet Astronomy Letters, 9, 12

Sobolev, A. M. 1986, Soviet Astronomy, 30, 399

Sobolev, A. M. 1989, Astron.Nachr., 310, 343

Sobolev, A. M. 1993, LNP, 412, 215

Sobolev, A. M. \& Deguchi, S. 1994a, ApJ, 433, 719

Sobolev, A. M. \& Deguchi, S. 1994b, Astron. Astrophys., 291, 569

Sobolev, A. M., Cragg, D. M., \& Godfrey, P. D. 1997, Astron. Astrophys., 324, 211

Sobolev, A. M., Cragg, D. M., \& Godfrey, P. D. 1997, MNRAS (Letters), 288, 39

Sobolev, A. M., Wallin, B. K., \& Watson, W. D. 1998, ApJ, 498, 763

Sobolev, A. M., Ostrovskii, A. B., Malyshev, A. V., et al. 2002, IAU Symposium, 206, 179

Sobolev, A. M., Watson, W. D., \& Okorokov, V. A. 2003, ApJ, 590, 333

Sobolev, A. M., Sutton, E. C., Cragg, D. M., \& Godfrey, P. D. 2005, Astrophys.Space Sci., 295, 189

Sobolev, A. M., Cragg, D. M., Ellingsen, S. P., et al. 2007, IAUS, 242, 81

Sobolev, A. M., Sutton, E. C., Watson, W. D., et al. 2008, Radiophys. and Radioastron., 13, S76

Strelnitskii, V. S. 1988, Proc.IAU Symp., 129, 239

Sutton, E. C., Sobolev, A. M., Ellingsen, S. P., et al. 2001, ApJ, 554, 173

van der Walt, D. J., Sobolev, A. M., \& Butner, H. 2007, Astron. Astrophys., 464, 1015

Vinsome, P. K. W. 1976, 4th SPE Symp. on Reservoir Simul. Soc. of Petroleum Engineers, 4, 149

Voronkov, M. A., Austin, M. C., \& Sobolev, A. M. 2002, Astron. Astrophys., 387, 310

Voronkov, M. A., Sobolev, A. M., Ellingsen S. P., et al. 2005, Astrophys.Space Sci., 295, 217

Wallin, B. K., Watson, W. D., \& Wyld, H. W. 1998, ApJ, 495, 774

Watson, W. D. \& Wyld, H. W. 2003, ApJ, 598, 357

Watson, W. D., Wiebe, D. S., McKinney, J. C., \& Gammie, C. F. 2004, ApJ, 604, 707

Xu, Y., Reid, M. J., Zheng, X. W., \& Menten, K. M. 2006, Science, 311, 54

Yates, J. A., Field, D., \& Gray, M. D. 1997, MNRAS, 285, 303

Yun, Y. J., Park, Y.-S., \& Lee, S. H. 2009, Astron. Astrophys., 507, 1785 